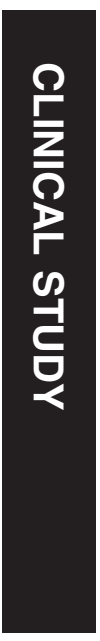

\title{
Patterns of adherence to NICE Glaucoma Guidance in two different service delivery models
}

${ }^{1}$ Manchester Royal Eye Hospital and Manchester Academic and Health Science Centre, Central Manchester University Hospitals NHS Foundation Trust, Manchester, UK

${ }^{2}$ Stepping Hill Hospital, Stockport, UK

Correspondence: A Chawla, Manchester Royal Eye Hospital and Manchester Academic and Health Science Centre, Central Manchester University Hospitals NHS Foundation Trust, Manchester M13 9WL, UK Tel: +44 (0)780947023; Fax: +44 (0)151 493 1420; E-mail: anand001@ hotmail.com

Received: 3 October 2011 Accepted in revised form: 24 April 2012

Published online: 31 August 2012

Presented at the Royal College of Ophthalmologist Congress-Birmingham 2011

\begin{abstract}
Purpose To assess adherence patterns to the UK National Institute of Health and Clinical Excellence (NICE) guidelines on glaucoma management (2009) in a tertiary referral centre shared care setting and in a district general hospital (DGH) setting.

Method We performed a retrospective case note analysis of $\mathbf{2 0 0}$ patients from two centres between January and June 2010. The two centres involved were a consultant-guided teaching hospital optometry-led shared care setting (setting 1) and a consultant-led DGH clinic setting (setting 2 ). The main outcome measures were compliance with eight of the main NICE guidelines on glaucoma diagnosis and management (2009).

Results Both centres showed good adherence to the guidelines regarding the choice of initial treatment $(96 \%$ vs $100 \%$, settings 1 and 2, respectively) and arranging appropriate monitoring intervals $(92 \%$ vs $86 \%$ ). However, significant differences were seen when assessing whether an optic disc image was obtained at the initial visit $(74 \%$ vs $10 \%$ ), whether an appropriate initial assessment was performed ( $96 \%$ vs $58 \%)$, whether patients' review interval complied with the NICE guidance regardless of hospital cancellations ( $92 \%$ vs $66 \%$ ), and whether concordance with medication was checked (88\% vs 24\%) (settings 1 and 2, respectively, $\boldsymbol{P}<0.01-$ Fisher's exact test). Conclusion Our study provides evidence to suggest that a hospital-based shared care service with trained optometrists using assessment sheets compares favourably to non-specialist glaucoma care delivered by ophthalmologists.
\end{abstract}

A Chawla ${ }^{1}$, I Patel ${ }^{1}$, C Yuen ${ }^{2}$ and C Fenerty

Eye (2012) 26, 1412-1417; doi:10.1038/eye.2012.171; published online 31 August 2012

Keywords: glaucoma; shared care; optometry

\section{Introduction}

The UK National Institute of Health and Clinical Excellence (NICE) recently published its guidance on the management of glaucoma with the aim of providing the best quality of care for glaucoma patients (April 2009). ${ }^{1}$ The scope of this document was to provide guidance on the diagnosis and management of chronic open-angle glaucoma and ocular hypertension and provide recommendations on the best way to manage such patients. A few months later, the National Patient Safety Agency issued an urgent alert to NHS trusts and other health-care providers to review their patient appointment systems and ensure that all patients with glaucoma are treated appropriately and on time. ${ }^{2}$ The implementation of the NICE guidelines is therefore essential.

Guidelines $^{3-5}$ have been developed to try and improve the quality of care for glaucoma patients but little work has been published to show the adherence to such guidelines. ${ }^{6}$ Such information can be useful to help units find ways to improve their compliance and in turn the quality of care for glaucoma patients. To our knowledge, no such work has been done with regard to adherence to the recent NICE guidance.

Increasing numbers of elderly patients with glaucoma have led to an overburdening of ophthalmic outpatient clinics in the United Kingdom. The role of optometrists in shared care schemes has led to much interest and 
research but the benefit of shared care systems still remains controversial. ${ }^{7-11}$

We therefore designed and carried out a study into how well NICE was being adhered to in two different settings: a consultant-guided teaching hospital optometry-led shared care setting (setting 1) and a consultant-led district general hospital (DGH) general clinic setting (setting 2). These two settings were chosen as both have a large workload of glaucoma suspects and low to moderate risk glaucoma patients. The aim of the study is first to see how well these units are adhering to the NICE guidance and second to evaluate the benefits of a shared care system in this context.

\section{Materials and methods}

Retrospective case note analysis of 200 patients from two centres: the Manchester Royal Eye Hospital optometryled shared care setting providing support to the wider hospital eye service (setting 1) and a DGH clinic setting providing secondary care to patients from two neighbouring counties in Stockport, Cheshire (population of 350000 ) and Buxton, Derbyshire (population of 27000) (setting 2). Patients were identified by obtaining hospital appointment lists of the appropriate clinics and consecutively choosing notes of glaucoma patients who met the inclusion criteria (see below). Using the NICE guidelines, we identified seven key standards for glaucoma and glaucoma suspects and designed a data collection sheet.

In setting 1, the optometrists, in this service, were all trained by glaucoma consultants and signed off as competent in variety of skills. In addition, all had, or were working towards, the Royal College of Optometrists Glaucoma Diploma parts A and B. They worked according to strict protocols with glaucoma assessment sheets forming part of the case record and acting as a prompt for collecting all relevant elements of the history, examination, and management plan. In setting 2, the clinic was run by one consultant, three middle grades, and a specialist registrar. They were working in a general clinic where both glaucoma and other patients with general ophthalmic problems were seen. The clinicians were not working to a specific protocol, rather they were carrying out 'conventional routine practice'. They had no specific training other than the Royal College Curriculum appropriate for their level.

\section{Setting 1}

We conducted a retrospective case note analysis of 100 consecutive glaucoma patients attending over a 2-month period-January to February 2010. This included 50 consecutive new patients from optometrist primary assessment clinic and 50 consecutive follow-up glaucoma patients seen in the optometry-led glaucoma assessment (OLGA) during this period. The data was collected by an ophthalmologist and an optometrist.

In the new patient group, only patients with glaucoma (primary open-angle glaucoma (POAG)/normal tension glaucoma (NTG)/pseudoexfoliative glaucoma (PXF)/ pigment dispersion glaucoma (PDS) or suspected glaucoma) were included in the data collection. In the follow-up group, only patients with a known diagnosis of glaucoma-either POAG/NTG/PXF/PDS were included. All other glaucomas were excluded.

The data was then entered into a Microsoft Excel spreadsheet and the results analysed.

\section{Setting 2}

We conducted a retrospective case note analysis of 100 glaucoma patients over a 6-month period-January to June 2010. This included 50 new patients and 50 followup patients seen in a glaucoma consultant-led general clinic at a time when the unit was working towards adherence to the NICE guidelines. The data was collected by one ophthalmologist.

All patient inclusion criteria were the same as in setting 1 and data were entered onto a standardised spreadsheet. We used Fisher's exact test to compare the two samples. Stata software (version 7.0; Stata Corp., College Station, TX, USA) was used to carry out the data analyses.

\section{Standards}

Standard 1: At diagnosis patients have:

- Goldmann applanation tonometry

- Central corneal thickness

- Gonioscopy

- Disc assessment

- Visual field assessment

Standard 2: An optic nerve head image is obtained at diagnosis for baseline documentation.

Standard 3: Choice of treatment and drug used follows the NICE algorithm.

Standard 4: At each monitoring visit, patients have Goldmann tonometry/disc assessment and van Herick AC depth assessment. ${ }^{12,13}$

Standard 5: Patients' planned review intervals are set in accordance with the NICE algorithm.

Standard 6: Patients' actual review interval complies with the NICE guidance regardless of hospital cancellations. 
Standard 7: Patients' adherence to treatment and eye drop instillation technique is checked at review appointments.

\section{Results}

Data from 200 patients were collected (100 from setting 1 and 100 from setting 2). The results are summarised in Tables 1 and 2. There was no significant difference between the mean age of patients in each setting; in the new patient group, the mean age was $58.9 \pm 11.2$ (mean $\pm \mathrm{SD}$ ) in setting 1 and $65.1 \pm 12.8$ (mean $\pm \mathrm{SD}$ )

Table 1 Adherence to the NICE guidelines in optometry-led shared care system and DGH clinic setting (new patients)

\begin{tabular}{lccc}
\hline & $\begin{array}{c}\text { Setting 1: } \\
\text { optometry } \\
\text { shared care } \\
(\mathrm{n}=50)\end{array}$ & $\begin{array}{c}\text { Setting 2: } \\
\text { DGH } \\
(\mathrm{n}=50)\end{array}$ & P-value \\
\hline Standards & & & \\
\hline $\begin{array}{l}\text { Initial assessment } \\
\quad \text { Goldmann applantion }\end{array}$ & $100 \%$ & $100 \%$ & \\
$\quad$ tonometry & $96 \%$ & $58 \%$ & $<0.01$ \\
$\quad \begin{array}{l}\text { Central corneal thickness } \\
\text { Gonioscopy }\end{array}$ & $96 \%$ & $74 \%$ & $<0.01$ \\
$\begin{array}{l}\text { Disc assessment } \\
\quad \text { Visual field assessment }\end{array}$ & $100 \%$ & $100 \%$ & \\
& $100 \%$ & $100 \%$ & \\
$\begin{array}{l}\text { Obtain an optic nerve head } \\
\text { image diagnosis for baseline }\end{array}$ & $74 \%$ & $10 \%$ & $<0.01$ \\
$\begin{array}{l}\text { documentation } \\
\text { Choice of treatment and drug }\end{array}$ & $96 \%$ & $100 \%$ & \\
used follow the NICE algorithm & & & \\
\hline
\end{tabular}

Table 2 Adherence to the NICE guidelines in optometry-led shared care system and DGH clinic setting (follow-up patients)

\begin{tabular}{lccc}
\hline & $\begin{array}{c}\text { Setting 1: } \\
\text { optometry } \\
\text { shared care } \\
(\mathrm{n}=50)\end{array}$ & $\begin{array}{c}\text { Setting 2: } \\
\text { DGH } \\
(\mathrm{n}=50)\end{array}$ & P-value \\
Standards & & & \\
\hline $\begin{array}{l}\text { At each monitoring visit } \\
\quad \text { Goldmann tonometry }\end{array}$ & $100 \%$ & $100 \%$ & \\
$\quad \begin{array}{l}\text { Disc assessment } \\
\text { AC depth assessment }\end{array}$ & $100 \%$ & $90 \%$ & $<0.01$ \\
& $94 \%$ & $8 \%$ & $<0.01$ \\
$\begin{array}{l}\text { Planned review intervals } \\
\text { in accordance with the }\end{array}$ & $92 \%$ & $86 \%$ & \\
$\begin{array}{l}\text { NICE algorithm } \\
\text { Actual review intervals }\end{array}$ & $92 \%$ & $66 \%$ & $<0.01$ \\
in accordance with the & & & \\
$\begin{array}{l}\text { NICE algorithm regardless } \\
\text { of hospital cancellations }\end{array}$ & & & \\
$\begin{array}{l}\text { Patient's adherence to } \\
\text { treatment checked }\end{array}$ & $88 \%$ & $24 \%$ & $<0.01$ \\
\hline
\end{tabular}

in setting 2. In the follow-up group, the mean age was $67.3 \pm 12.4$ (mean \pm SD) in setting 1 and $74.4 \pm 12.0$ (mean $\pm \mathrm{SD}$ ) in setting 2 . The sex distribution was also similar between the two groups; in the new patient group, $50 \%$ were female in setting 1 and $52 \%$ were female in setting 2 . In the follow-up group, $52 \%$ were female in setting 1 and $54 \%$ were female in setting 2 . No statistical differences were seen in sex distribution. The most common diagnoses in the new patient group were glaucoma suspect (63\%), POAG (24\%), NTG (11\%), PDS (1\%), and PXF (1\%). In the follow-up group, the most common diagnoses were POAG (79\%), NTG (15\%), PXF (4\%), and PDS (2\%). The distribution of diagnosis was similarly distributed between the two settings.

In standard 1 (initial assessment), 100\% of patients in setting 1 had Goldmann tonometry, disc assessment, and visual fields. In all, 96\% had gonioscopy and 96\% had central corneal thickness measured. Similarly, in setting 2, $100 \%$ of patients had Goldmann tonometry, disc assessment, and visual fields, but only $74 \%$ had gonioscopy $(P<0.01)$ and only $58 \%$ had central corneal thickness $(P<0.01)$.

In standard 2 (obtaining optic nerve head image), setting 1 obtained an image in $74 \%$ of cases as compared with $10 \%$ in setting $2(P<0.01)$.

Standard 3 (initial treatment following the NICE algorithm) was adhered in $96 \%$ in setting 1 and $100 \%$ in setting 2.

Standard 4 (monitoring visit assessment): both settings performed applanation tonometry in all patients at their review visit. Disc assessment was performed in $100 \%$ of patients in setting 1 but only in $90 \%$ of the setting 2 patients $(P<0.01)$. Anterior chamber depth assessment was performed in $94 \%$ of patients in setting 1 but only in $8 \%$ of patients in setting $2(P<0.01)$.

Standard 5 (patients monitored in accordance to the NICE algorithm): this standard was complied with in $92 \%$ of patients in setting 1 and $86 \%$ of patients in setting 2 .

When looking into hospital cancellations (standard 6), setting 1 showed a $92 \%$ adherence to the NICE monitoring intervals despite of hospital cancellations, whereas setting 2 showed a $70 \%$ adherence to this standard $(P<0.01)$.

Standard 7 (checking compliance) showed that setting 1 checked compliance in $88 \%$ of its follow-up glaucoma patients. In setting 2 , this was only adhered to in $24 \%$ of patients $(P<0.01)$.

\section{Discussion}

There has been much debate into the role of shared care systems in the management of glaucoma. ${ }^{7-11}$ The Manchester OLGA clinic manages mainly low- and moderate-risk glaucoma cases, and patients with ocular 
hypertension are suspected of having glaucoma. The consultant-led clinics in Manchester Royal Eye Hospital predominantly manage more complex glaucoma cases and the service provided in these two setting is not immediately comparable. A consultant-led clinic in a DGH with a larger proportion of early and moderate glaucoma cases has a more comparable workload and was used for comparison in this study.

Our study provides evidence to suggest that a hospital shared care service with trained optometrists has higher rates of adherence to the NICE guidelines, as compared with non-specialist glaucoma care delivered by ophthalmologists. Specifically, the study highlights the advantage of using pre-designed assessment sheets in driving shared care schemes in glaucoma management. The use of glaucoma by trained optometrists also has benefits in terms of reliability and accuracy of measurements, which has been assessed in previous studies. ${ }^{10,13,14}$

The results from four of the guidelines show a statistically significant improved performance in setting 1: the optometrists shared care setting as compared with setting 2: the DGH clinic setting. To our knowledge, no similar comparable studies have been previously published and so we cannot compare with experiences from other units.

The only comparable indicator to previous studies was the rate of gonioscopy performed by community optometrists. In our optometry cohort, $96 \%$ performed gonioscopy, which is much higher than stated in previous community optometry studies (ranging from $0-53 \%) .{ }^{15,16}$ This may be explained by the fact that the hospital-based optometrists in this scheme are fully trained in gonioscopy, which is less common for community optometrists. Similarly, the results for visual field testing were much higher in our study as compared with a previous study looking at community optometrists. ${ }^{15}$ Such improvements are encouraging and may reflect the impact of the NICE guidance and improved knowledge and education.

Sub-group examination revealed important differences in the way glaucoma was managed in the two settings. In setting 1, the optometrists used pre-designed glaucoma collection sheets for all new and follow-up patients, whereas in setting 2, the ophthalmologists in the DGH clinic were variable in their use of a new-patient glaucoma assessment sheet. Second, the optometrists in setting 1 managed their own appointment system and rearranged appointments when cancellations arose.

Standards 1 and 4 highlight the advantages of having pre-designed glaucoma assessment sheets. The optometry-led service performed significantly better than the DGH setting because all new patients had CCT and gonioscopy performed and all follow-up patients had anterior chamber depth assessment. The data set of the assessment sheets for the optometry-led services includes each of these elements and clearly acts as an aide memoir for the assessor.

The results from standards 5 and 6, which assess whether patients are followed up at the appropriate time interval despite hospital cancellations, highlight one of the major advantages of the optometry-led shared care service. In all, $92 \%$ of patients in the OLGA service were followed up during the appropriate time interval. Of these, no patients' appointments were delayed because of hospital cancellations. In the DGH setting, only $66 \%$ of patients were followed up during the appropriate time interval. In addition to reflecting the NICE guidance, this standard also relates to the NPSA recommendations regarding cancellations of glaucoma patient's follow-up appointments. The problem of demand-capacity mismatch is understandable when referral guidelines are widened and dominated by non-contact tonometry falsepositives and is a problem nationwide. Shared care promises to increase patient access to eye health-care services and thereby enhance continuity and quality of care. ${ }^{17,18}$

The worst performing indicator in both settings was obtaining disc imaging on initial visit, although the optometry-led service showed a higher compliance than the DGH setting ( $74 \%$ vs $10 \% P<0.01)$. A common problem in both systems was access to disc imaging at the time of patients' attendances; the DGH setting involved a clinic not set up with a dedicated disc imaging facility. Furthermore, there was no dedicated section on the optometry-led service assessment sheet for data collection on disc imaging. This further supports the value of assessment sheets in assessing and monitoring glaucoma. We have now changed the glaucoma assessment sheet, in our department, to include a section for disc imaging.

NICE has stressed the importance of checking for concordance and adverse effects from medication. We found that in the optometry-led service compliance was checked in $88 \%$ of patients, whereas compliance was only checked in $24 \%$ of patients in the DGH group. Again the optometry-led service assessment sheet has a section to record compliance, whereas no such form exists in our DGH setting. In the DGH setting, there is now a fully independent glaucoma practitioner clinic in which drop instillation techniques, patient education, and compliance with medications are assessed. There is also a well-established glaucoma patient support group run by the glaucoma consultant and his glaucoma practitioner, which provides extra patient education and support and has received very positive feedback from patient groups.

It is important to appreciate that such a shared care setting needs appropriate consultant support and input. 
In our service, the optometry-led shared care service is managed by two glaucoma consultants who have been responsible for setting up the scheme, designing assessment and management protocols, and recruiting and training optometrists. The ultimate clinical responsibility for the glaucoma patients still lies with the consultant ophthalmologist. We must also make it clear that this study has not assessed reliability of measurements, which has been addressed in previous studies. ${ }^{8-10}$

The study shows that a streamlined glaucoma service whereby all individuals have an up to date knowledge of the NICE guidance improves the quality of glaucoma care. Having well-designed assessment sheets for all new and follow-up glaucoma patients serves as a checklist to ensure that all patients are assessed and managed appropriately. The advantage of developing assessment tools based on the national guidelines has been studied in Cancer Screening programmes. ${ }^{18}$ As well as ensuring adherence to guidelines, they minimise differences in provider practices and maximise the performance of the service. ${ }^{19}$

Our study provides evidence for the use of Optometryled shared care to improve the quality of glaucoma management using the NICE guidance as the reference standard. Following on from a previous study by Vernon et al., ${ }^{16}$ we hope that the information from our scheme will be of value to those departments considering investing in shared care scheme.

To our knowledge, this is the first study that has compared adherence with the NICE guidance in two different settings; thus allowing us to identify ways to help other units improve their standards of care. The key feature is of having a hands-on management role for the consultant clinical lead with well-designed assessment sheets. Such assessment sheets are more likely to be followed in dedicated clinics.

\section{Summary}

\section{What was known before}

- Many guidelines have been developed to try and improve the quality of care for glaucoma patients, but very little work has been published to show the adherence to such guidelines. Shared care systems in ophthalmology are in place in a few units throughout the country.

- The effectiveness of such systems still remains controversial.

What this study adds

- Our study shows good adherence to the new NICE guidelines in two different settings.

- Our study provides evidence to suggest that a hospitalbased shared care service with trained optometrists compares favourably to non-specialist glaucoma care delivered by ophthalmologists.

\section{Conflict of interest}

The authors declare no conflict of interest.

\section{References}

1 UK National Institute of Health and Clinical Excellence Glaucoma. Diagnosis and management of chronic open angle glaucoma and ocular hypertension 2009 (accessed from http://guidance.nice.org.uk/CG85/ Guidance/pdf/English).

2 National Patient Safety Agency. Preventing delay to followup for patients with glaucoma 2009 (accessed from http:// www.nrls.npsa.nhs.uk/resources/?EntryId45 =61908).

3 American Academy of Ophthalmology. Preferred practice pattern-primary open angle glaucoma 2010 (accessed from http://one.aao.org/CE/PracticeGuidelines/ PPP Content.aspx? $\mathrm{cid}=$ 93019a87-4649-4130-8f94b6a9b19144d2).

4 European Glaucoma Society. Terminology and Guidelines for Glaucoma, 3rd edition 2008 (accessed from http:// www.eugs.org/eng/EGS_guidelines.asp).

5 The Royal College of Ophthalmologists. Guidelines for management of Glaucoma and Ocular Hypertension 2004 (accessed from http://www.mrcophth.com/ glaucoma2004.pdf).

6 Vernon SA, Ghosh G. Do locally agreed guidelines for optometrists concerning the referral of glaucoma suspects influence referral practice? Eye 2001; 15: 458-463.

7 Hitchings RA. Shared care for glaucoma. Br J Ophthalmol 1995; 79: 626.

8 Henson DB, Spencer AF, Harper R, Cadman EJ. Community refinement of glaucoma referrals. Eye 2003; 17: 21-26.

9 Banes MJ, Culham MC, Brunce C, Xing X, Viswanathan A, Garway-Heath D. Agreement between optometrists and ophthalmologists on clinical management decisions for patients with glaucoma. $\mathrm{Br} J$ Ophthalmol 2006; 90: 579-585.

10 Spry PGD, Spencer ID, Sparrow JM, Peters PJ, Brookes ST, Gray $\mathrm{S}$ et al. The Bristol shared care glaucoma study: reliability of community optometric and hospital eye services test measures. Br J Ophthalmol 1999; 83: 707-712.

11 Gray SF, Spry PGD, Brookes ST, Peters TJ, Spencer IC, Baker IA et al. The Bristol Shared Care Glaucoma Study: outcome at follow up at 2 years. Br J Ophthalmol 2000; 84: 456-463.

12 van Herick W, Shaffer RN, Schwartz A. Estimation of width of angle of anterior chamber incidence and significance of the narrow angle. Am J Ophthalmol 1969; 68: 626-629.

13 Ho S, Vernon SA. Decision making in chronic glaucoma optometrists vs ophthalmologists in a shared care service. Ophthalmic Physiol Opt 2011; 31: 168-173.

14 Bourne RR, French KA, Chang L, Borman AD, Hingorani M, Newsom WD. Can a community optometrist-based referral refinement scheme reduce false-positive glaucoma hospital referrals without compromising quality of care? The Community And Hospital Allied Network Glaucoma Evaluation Scheme (CHANGES). Eye (Lond) 2010; 24(5): 881-887.

15 Shah R, Edgar DF, Spry PG, Harper RA, Kotecha A, Rughani $S$ et al. Glaucoma detection: the content of optometric eye examinations for a presbyopic patient of African racial descent. Br J Ophthalmol 2009; 93: 492-496. 
16 Vernon SA, Adair A. Shared care in glaucoma: a national study of secondary care lead schemes in England. Eye 2010; 24: 265-269.

17 Lu CY, Lu VH, Goldberg I, Day RO. Towards integrated care: Australia's new model of care for patients with glaucoma. Med J Aust 2010; 193(4): 200-201.

18 Mandalos A, Bourne R, French K, Newsom W, Chang L. Shared care of patients with ocular hypertension in the
Community and Hospital Allied Network Glaucoma Evaluation Scheme (CHANGES). Eye (Lond) 2012; 26(4): 564-567.

19 Wieland DL, Reimers LL, Wu E, Nathan LM, Gruenberg T, Abadi $\mathrm{M}$ et al. Performance of implementing guidelinedriven cervical cancer screening measures in an inner-city hospital system. J Low Genit Tract Dis 2011; 15(4): 296-302. 